\title{
COMPARISON PRINCIPLE FOR A NONLINEAR PARABOLIC PROBLEM OF A NONMONOTONE TYPE
}

Abstract. A nonlinear parabolic problem with the Newton boundary conditions and its weak formulation are examined. The problem describes nonstationary heat conduction in inhomogeneous and anisotropic media. We prove a comparison principle which guarantees that for greater data we obtain, in general, greater weak solutions. A new strategy of proving the comparison principle is presented.

1. Introduction. Comparison principles are important features of second order partial differential equations. They hold for linear and many nonlinear problems of elliptic and parabolic types. It seems that the ellipticity of an appropriate operator is the main feature which assures the comparison principle (compare [5, 10]).

In this paper we deal with the following quasilinear parabolic problem: Find $u(x, t) \in C^{1}(\bar{Q}) \cap C^{2}(Q)$ such that

$$
\begin{aligned}
c(x, t) \varrho(x, t) \partial_{t} u-\operatorname{div}(\mathcal{A}(x, t, u) \nabla u) & =f(x, t) & & \text { in } Q, \\
\alpha u+n^{\top} \mathcal{A}(x, t, u) \nabla u & =g(x, t) & & \text { on } \Sigma, \\
u(x, 0) & =u_{0}(x) & & \text { in } \Omega, t=0,
\end{aligned}
$$

where $\Omega \subset \mathbb{R}^{d}, d=1,2, \ldots$, is a bounded domain with Lipschitz continuous boundary, $I=[0, T], T>0, Q=\Omega \times I, \Sigma=\partial \Omega \times I, n=\left(n_{1}, \ldots, n_{d}\right)^{\top}$ is the outward unit normal to $\partial \Omega$, the symbol ${ }^{\top}$ means transposition, $\mathcal{A}=$ $\left(a_{i j}\right)_{i, j=1}^{d}$ is a uniformly positive definite matrix with respect to all variables, $c>0, \varrho>0$ in $\bar{Q}$ and $\alpha \geq 0$ on $\Sigma$. The symbol $\partial_{t}$ denotes the time derivative $\partial / \partial t, \bar{Q}$ stands for the closure of the set $Q, C^{1}(\bar{Q})$ is the space of functions with first derivatives continuous in $\bar{Q}$ and similarly $C^{2}(Q)$ is the space of

2000 Mathematics Subject Classification: Primary 35K55.

Key words and phrases: evolution heat conduction problem, Newton boundary conditions, comparison principle. 
functions with all the second derivatives continuous in $Q$. For the time being let the functions $c, \varrho, \mathcal{A}, \alpha, f, g$ and $u_{0}$ be sufficiently smooth.

Problem (1.1) describes, e.g., time dependent heat conduction in nonlinear inhomogeneous and anisotropic media. The unknown function $u$ represents the distribution of temperature in the domain $\Omega$, which is supposed to be filled with some inhomogeneous and anisotropic material. The properties of this material are described by the heat capacity $c$, mass density $\varrho$ and by the matrix $\mathcal{A}$ of heat conductivities. The function $f$ represents volume heat sources. The symbols $\alpha$ and $g$ denote the heat transfer coefficient and the density of surface heat sources, respectively.

In Section 3 we prove a comparison principle for weak solutions of the nonlinear parabolic problem (1.1), which is nonmonotone and in general nonpotential. We shall see that a direct consequence of the comparison principle is the uniqueness of weak solutions. Our proof of the comparison principle is a generalization of that in [8], where an elliptic boundary value problem is studied. Similar results are obtained in another way in [1], where different boundary conditions are examined.

Many heat conduction problems in technical practice involve a nonlinear dependence of the heat conduction coefficient on the temperature itself. This dependence always leads to a nonmonotone operator. We examine this fact in $[11]$.

2. Weak formulation. In what follows, all equalities and inequalities for functions defined on $\Omega, Q, \Sigma, \partial \Omega, \ldots$ have to be considered up to a set of zero measure. Note that $C, C_{1}, C_{2}, \ldots$ are the so called generic constants and they can represent different values in different occurrences.

From now on we assume, without loss of generality, that $c=1$ and $\varrho=1$ in $Q$ and that $\mathcal{A}=\mathcal{A}(x, t, r)$ and $\alpha=\alpha(x, t)$ are bounded measurable functions, i.e., there exists a constant $C$ such that

$$
\underset{x, t, r, i, j}{\operatorname{ess} \sup }\left|a_{i j}(x, t, r)\right| \leq C, \quad \operatorname{essup}|\alpha(s, t)| \leq C,
$$

where $x \in \Omega, t \in I, r \in \mathbb{R}, i, j \in\{1, \ldots, d\}$, and $s \in \partial \Omega$. Moreover, we suppose that $\mathcal{A}(x, t, \cdot)$ is continuous for almost every $(x, t) \in Q$, i.e., $\mathcal{A}$ satisfies the Carathéodory conditions. The components $a_{i j}$ are considered to be Lipschitz continuous with respect to the last variable, i.e., there exists $C_{L}>0$ such that for all $r_{1}, r_{2} \in \mathbb{R}$ and for almost all $x \in \Omega$ and $t \in I$ we have

$$
\left|a_{i j}\left(x, t, r_{1}\right)-a_{i j}\left(x, t, r_{2}\right)\right| \leq C_{L}\left|r_{1}-r_{2}\right|, \quad i, j=1, \ldots, d .
$$

Further, let there exist $C_{0}>0$ such that for almost all $x \in \Omega$ and $t \in I$,

$$
C_{0} \eta^{\top} \eta \leq \eta^{\top} \mathcal{A}(x, t, r) \eta \quad \forall r \in \mathbb{R}, \forall \eta \in \mathbb{R}^{d}
$$


and let $0 \leq \alpha(s, t)$ for almost all $(s, t) \in \Sigma$. Moreover, we assume that there exists a constant $\alpha_{0}>0$ and a nonempty relatively open subset $\Gamma \subset \partial \Omega$ with meas $\Gamma>0$ such that

$$
\alpha(s, t) \geq \alpha_{0} \quad \text { for almost all } s \in \Gamma \text { and almost all } t \in I .
$$

We will usually write only $\mathcal{A}(u), f, g, \ldots$ instead of $\mathcal{A}(x, t, u), f(x, t)$, $g(x, t), \ldots$, i.e., the dependence on the space variable $x$ and time variable $t$ will not be explicitly indicated, since no ambiguity can occur.

We assume that $f \in L^{2}(Q), g \in L^{2}(\Sigma)$, and $u_{0} \in L^{2}(\Omega)$. Let us set $V=H^{1}(\Omega), \mathcal{H}=L^{2}(\Omega)$ and denote the dual space by a star, e.g., $V^{*}=$ $\left(H^{1}(\Omega)\right)^{*}$. We see that $V \subset \mathcal{H}$, and therefore $\mathcal{H}^{*} \subset V^{*}$. Moreover, these imbeddings are continuous. The symbol $L^{2}(I ; V)$ stands for the well-known Bochner space of square integrable mappings from the interval $I$ into $V$. This space is equipped with the norm

$$
\|v\|_{L^{2}(I ; V)}^{2}=\int_{0}^{T}\|v(t)\|_{V}^{2} d t .
$$

If $v=v(x, t)$, then $v(t)$ denotes the function defined on $\Omega$ such that $v(t)(x)=$ $v(x, t)$ for $x \in \Omega$ and $t \in I$. Let $V_{1}$ and $V_{2}$ be two Banach spaces. Then we put

$$
W\left(I ; V_{1}, V_{2}\right)=\left\{v \in L^{2}\left(I ; V_{1}\right) \mid \partial_{t} v \in L^{2}\left(I ; V_{2}\right)\right\} .
$$

We have the inclusion $W\left(I ; V, V^{*}\right) \subset C(I ; \mathcal{H})$ (see for instance [4, p. 161]), where $C(I ; \mathcal{H})$ is the space of continuous mappings from $I$ into $\mathcal{H}$. Thus, the values of any function $v=v(x, t)$ from $W\left(I ; V, V^{*}\right)$ are well defined at all time levels $t \in I$, i.e., $v(t) \in \mathcal{H}$ has a meaning for all $t \in I$.

To shorten the weak formulation of problem (1.1) we introduce the following notation:

$$
\begin{aligned}
a(t, y ; w, v) & =(\mathcal{A}(\cdot, t, y) \nabla w, \nabla v)_{0, \Omega}+(\alpha(\cdot, t) w, v)_{0, \partial \Omega}, \\
F(t, v) & =(f(\cdot, t), v)_{0, \Omega}+(g(\cdot, t), v)_{0, \partial \Omega},
\end{aligned}
$$

where $y, w, v \in H^{1}(\Omega), t \in I$ and the symbols $(\cdot, \cdot)_{0, \Omega}$ and $(\cdot, \cdot)_{0, \partial \Omega}$ stand for the inner products in the Lebesgue spaces $L^{2}(\Omega)$ and $L^{2}(\partial \Omega)$, respectively. Similarly we denote by $\|\cdot\|_{0, \Omega}$ and $\|\cdot\|_{0, \partial \Omega}$ the associated norms. Finally, $\|\cdot\|_{1, \Omega}$ stands for the norm in the Sobolev space $H^{1}(\Omega)$.

We can easily see from $(2.5)$ that the form $a(t, y ; w, v)$ is nonlinear in $t, y$ and linear in $w$ and $v$.

Definition 2.1. A function $u \in W\left(I ; V, V^{*}\right)$ is said to be a weak solution of problem (1.1) if

$$
V^{*}\left\langle\partial_{t} u, v\right\rangle_{V}+a(t, u ; u, v)=F(t, v)
$$


for all $v \in H^{1}(\Omega)$ and for almost every $t \in I$, and

$$
u(x, 0)=u_{0}(x) \quad \text { for almost every } x \in \Omega .
$$

Here the time derivative $\partial_{t} u$ should be understood as an element of $L^{2}\left(I ; V^{*}\right)$, i.e., $\partial_{t} u(\cdot, t) \in\left(H^{1}(\Omega)\right)^{*}$ for almost every $t \in I$. The symbol $V^{*}\langle\cdot, \cdot\rangle_{V}$ in $(2.7)$ means the duality pairing between $V^{*}$ and $V$. Note that if $w^{*} \in \mathcal{H}^{*} \subset V^{*}$ and $v \in V \subset \mathcal{H}$, we have

$$
V^{*}\left\langle w^{*}, v\right\rangle_{V}=\mathcal{H}^{*}\left\langle w^{*}, v\right\rangle_{\mathcal{H}} .
$$

If $w \in \mathcal{H}$ then we define $w^{*} \in \mathcal{H}^{*}$ by $\mathcal{H}^{*}\left\langle w^{*}, y\right\rangle_{\mathcal{H}}=(w, y)_{\mathcal{H}}$ for all $y \in \mathcal{H}$, where $(\cdot, \cdot)_{\mathcal{H}}$ denotes the inner product in $\mathcal{H}$. By the Riesz theorem every continuous linear functional on $\mathcal{H}$ is of this form for a unique $w \in \mathcal{H}$. Thus, for any $w \in \mathcal{H}$, appropriate $w^{*} \in \mathcal{H}^{*}$ and any $v \in V$, we have

$$
(w, v)_{\mathcal{H}}=\mathcal{H}^{*}\left\langle w^{*}, v\right\rangle_{\mathcal{H}}=V^{*}\left\langle w^{*}, v\right\rangle_{V} .
$$

Therefore, if $u \in W(I ; V, \mathcal{H})$, i.e., $\partial_{t} u \in L^{2}(Q)$, then

$$
V^{*}\left\langle\partial_{t} u, v\right\rangle_{V}=\left(\partial_{t} u, v\right)_{\mathcal{H}}=\int_{\Omega} \partial_{t} u(x, t) v(x) d x
$$

for all $v \in H^{1}(\Omega)$.

From the uniform positive definiteness of the matrix $\mathcal{A}$ (see (2.3)) and from (2.4) we directly have an important property of the form $a$ : There exists a positive constant $C_{0}$ such that

$$
C_{0}\|v\|_{1, \Omega}^{2} \leq a(t, y ; v, v) \quad \forall y, v \in H^{1}(\Omega), \text { and for a.e. } t \in I .
$$

Later we will need the well known Friedrichs inequality in this form:

$$
\|v\|_{1, \Omega}^{2} \leq C\left(\|\nabla v\|_{0, \Omega}^{2}+\|v\|_{0, \Gamma}^{2}\right) \quad \forall v \in H^{1}(\Omega),
$$

where $\Gamma \subset \partial \Omega$ is an arbitrary relatively open set with meas $\Gamma>0$.

Before we introduce the comparison principle in Theorem 3.1, we make a few remarks about the existence of weak solutions of problem (2.7). There is an extensive literature where the problem of the existence of weak solutions of elliptic and parabolic problems is considered (for example $[9,4,3]$ ). With the aid of several techniques from these monographs it is possible to establish the existence of a weak solution of problem (2.7). We just note that the proof is based on the so-called pseudomonotony, because neither the theory of monotone operators nor the theory of potential operators can be applied (see $[6])$.

3. Comparison principle. First of all we mention that we have to assume weak solutions of (2.7) to be more regular. To be able to use (2.8) we consider weak solutions in $W(I ; V, \mathcal{H})$, which is a subspace of $W\left(I ; V, V^{*}\right)$. 
THEOREM 3.1. Let $u_{1}, u_{2} \in W(I ; V, \mathcal{H})$ be two weak solutions of problem (1.1) corresponding to $f_{1}, f_{2} \in L^{2}(Q), g_{1}, g_{2} \in L^{2}(\Sigma)$, and to the initial conditions $u_{01}, u_{02} \in L^{2}(\Omega)$, respectively. Assume that

$$
\begin{array}{cc}
f_{1} \geq f_{2} & \text { a.e. in } Q, \\
g_{1} \geq g_{2} & \text { a.e. in } \Sigma, \\
u_{01} \geq u_{02} & \text { a.e. in } \Omega .
\end{array}
$$

Then $u_{1} \geq u_{2}$ a.e. in $Q$.

Proof. Put $Q_{0}=\left\{(x, t) \in Q \mid u_{1}(x, t)<u_{2}(x, t)\right\}$ and assume, to the contrary, that

$$
\text { meas } Q_{0}>0 \text {. }
$$

For fixed $\varepsilon>0$ define

$$
Q_{\varepsilon}=\left\{(x, t) \in Q_{0} \mid u_{2}(x, t)-u_{1}(x, t)>\varepsilon\right\}
$$

(cf. Figure 1) and

$$
v_{\varepsilon}=v_{\varepsilon}(x, t)=\min \left(\varepsilon,\left(u_{2}-u_{1}\right)^{+}\right),
$$

where $u^{+}$denotes the positive part, i.e., $u^{+}=u$ if $u>0$ and $u^{+}=0$ if $u \leq 0$. Because $v \mapsto \min \left(\varepsilon, v^{+}\right)$maps $H^{1}(Q)$ into itself (see [6]), we have $v_{\varepsilon} \in H^{1}(Q)$. (Note that this mapping is continuous.) Consequently, $v_{\varepsilon}(\cdot, t) \in H^{1}(\Omega)$ for almost every $t \in I$ and therefore, it can be applied as a test function in (2.7). For a better understanding how $v_{\varepsilon}$ is defined we rewrite (3.5) as

$$
v_{\varepsilon}(x, t)= \begin{cases}\varepsilon & \text { in } Q_{\varepsilon}, \\ u_{2}(x, t)-u_{1}(x, t) & \text { in } Q_{0} \backslash Q_{\varepsilon}, \\ 0 & \text { in } Q \backslash Q_{0} .\end{cases}
$$

Subtracting the weak formulations (2.7) for $u_{1}$ and $u_{2}$ with $v_{\varepsilon}$ as a test function and employing (2.8), we obtain

$$
\begin{aligned}
& \left(\partial_{t}\left(u_{1}-u_{2}\right), v_{\varepsilon}\right)_{0, \Omega}+\left(\mathcal{A}\left(u_{1}\right) \nabla u_{1}-\mathcal{A}\left(u_{2}\right) \nabla u_{2}, \nabla v_{\varepsilon}\right)_{0, \Omega} \\
& \quad+\left(\alpha u_{1}-\alpha u_{2}, v_{\varepsilon}\right)_{0, \partial \Omega}=\left(f_{1}-f_{2}, v_{\varepsilon}\right)_{0, \Omega}+\left(g_{1}-g_{2}, v_{\varepsilon}\right)_{0, \partial \Omega} .
\end{aligned}
$$

Since $v_{\varepsilon} \geq 0, \alpha \geq 0, u_{1}<u_{2}$ in $Q_{0}$ and $\operatorname{supp} v_{\varepsilon} \subset Q_{0}$, we have

$$
\left(\alpha u_{1}-\alpha u_{2}\right) v_{\varepsilon} \leq 0 \quad \text { on } \Sigma=\partial \Omega \times I .
$$

Note that $v_{\varepsilon}(\cdot, 0)=0$ a.e. in $\Omega$ (see (3.3)). Hence, using the Fubini theorem, 
integration by parts, and (3.6), we have

$$
\begin{aligned}
\int_{Q} \partial_{t}\left(u_{1}-u_{2}\right) v_{\varepsilon} d x d t=\int_{\Omega} \int_{0}^{T} \partial_{t}\left(u_{1}-u_{2}\right) v_{\varepsilon} d t d x \\
=-\int_{\Omega} \int_{0}^{T}\left(u_{1}-u_{2}\right) \partial_{t} v_{\varepsilon} d t d x+\int_{\Omega}\left(u_{1}(T)-u_{2}(T)\right) v_{\varepsilon}(T) d x-0 \\
=\int_{Q_{0} \backslash Q_{\varepsilon}} v_{\varepsilon} \partial_{t} v_{\varepsilon} d x d t+\int_{\Omega}\left(u_{1}(T)-u_{2}(T)\right) v_{\varepsilon}(T) d x \\
=\int_{Q_{0} \backslash Q_{\varepsilon}} \frac{1}{2} \partial_{t} v_{\varepsilon}^{2} d x d t+\int_{\Omega}\left(u_{1}(T)-u_{2}(T)\right) v_{\varepsilon}(T) d x \\
=\int_{\Omega}^{T} \int_{0} \frac{1}{2} \partial_{t} v_{\varepsilon}^{2} d t d x+\int_{\Omega}\left(u_{1}(T)-u_{2}(T)\right) v_{\varepsilon}(T) d x \\
=\int_{\Omega}^{1} \frac{1}{2} v_{\varepsilon}^{2}(T) d x-0+\int_{\Omega}\left(u_{1}(T)-u_{2}(T)\right) v_{\varepsilon}(T) d x \\
=\int_{\Omega_{0}(T)}\left[\frac{1}{2} v_{\varepsilon}(T)-\left(u_{2}(T)-u_{1}(T)\right)\right] v_{\varepsilon}(T) d x \leq 0,
\end{aligned}
$$

where $\Omega_{0}(T)=\left\{x \in \Omega \mid u_{1}(x, T)<u_{2}(x, T)\right\}$ is indicated in Figure 1. The last inequality of (3.9) holds, because $v_{\varepsilon}(T) \leq u_{2}(T)-u_{1}(T)$ in $\Omega_{0}(T)$.

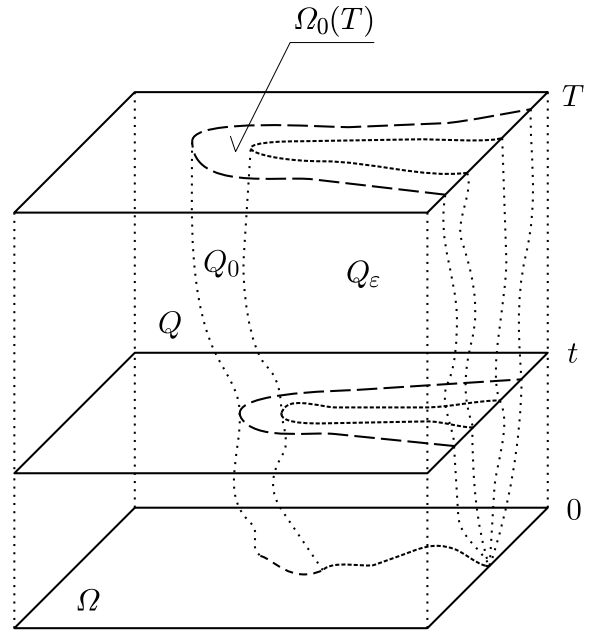

Fig. 1 
Using now the fact that $\mathcal{A}$ is uniformly positive definite (see (2.3)), the definition of $v_{\varepsilon}$ (see (3.6)), (3.7) integrated over $I,(3.9),(3.8),(3.1)$ and (3.2), we obtain

$$
\begin{aligned}
C_{0}\left\|\nabla v_{\varepsilon}\right\|_{0, Q}^{2} \leq & \int_{0}^{T}\left(\mathcal{A}\left(u_{1}\right) \nabla v_{\varepsilon}, \nabla v_{\varepsilon}\right)_{0, \Omega} d t \\
= & \left(\mathcal{A}\left(u_{1}\right) \nabla\left(u_{2}-u_{1}\right), \nabla v_{\varepsilon}\right)_{0, Q_{0} \backslash Q_{\varepsilon}} \\
= & \left(\mathcal{A}\left(u_{1}\right) \nabla u_{2}-\mathcal{A}\left(u_{1}\right) \nabla u_{1}, \nabla v_{\varepsilon}\right)_{0, Q} \\
= & \left(\mathcal{A}\left(u_{1}\right) \nabla u_{2}-\mathcal{A}\left(u_{2}\right) \nabla u_{2}, \nabla v_{\varepsilon}\right)_{0, Q} \\
& +\left(\partial_{t}\left(u_{1}-u_{2}\right), v_{\varepsilon}\right)_{0, Q}+\left(\alpha u_{1}-\alpha u_{2}, v_{\varepsilon}\right)_{0, \Sigma} \\
& +\left(f_{2}-f_{1}, v_{\varepsilon}\right)_{0, Q}+\left(g_{2}-g_{1}, v_{\varepsilon}\right)_{0, \Sigma} \\
\leq & \left(\left(\mathcal{A}\left(u_{1}\right)-\mathcal{A}\left(u_{2}\right)\right) \nabla u_{2}, \nabla v_{\varepsilon}\right)_{0, Q} .
\end{aligned}
$$

We now apply the Cauchy-Schwarz inequality and the Lipschitz continuity of the entries of the matrix $\mathcal{A}$, which is assumed in (2.2), to obtain

$$
\begin{aligned}
\left(\left(\mathcal{A}\left(u_{1}\right)-\mathcal{A}\left(u_{2}\right)\right)\right. & \left.\nabla u_{2}, \nabla v_{\varepsilon}\right)_{0, Q_{0} \backslash Q_{\varepsilon}} \\
& \leq\left\|\left(\mathcal{A}\left(u_{1}\right)-\mathcal{A}\left(u_{2}\right)\right) \nabla u_{2}\right\|_{0, Q_{0} \backslash Q_{\varepsilon}}\left\|\nabla v_{\varepsilon}\right\|_{0, Q_{0} \backslash Q_{\varepsilon}} \\
& \leq \varepsilon C\left\|\nabla u_{2}\right\|_{0, Q_{0} \backslash Q_{\varepsilon}}\left\|\nabla v_{\varepsilon}\right\|_{0, Q} .
\end{aligned}
$$

Combining (3.10) and (3.11), we have

$$
\left\|\nabla v_{\varepsilon}\right\|_{0, Q} \leq \varepsilon C\left\|\nabla u_{2}\right\|_{0, Q_{0} \backslash Q_{\varepsilon}} .
$$

The fact that $v_{\varepsilon} \leq u_{2}-u_{1}$ on $Q_{0}$ and inequality (2.4) yield

$$
0 \leq v_{\varepsilon} \leq u_{2}-u_{1} \leq \frac{\alpha}{\alpha_{0}}\left(u_{2}-u_{1}\right) \quad \text { on } \Gamma \cap Q_{0} \text { for a.e. } t \in I,
$$

and thus

$$
v_{\varepsilon}^{2} \leq \frac{\alpha}{\alpha_{0}}\left(u_{2}-u_{1}\right) v_{\varepsilon} \quad \text { on } \Gamma \text {, for a.e. } t \in I .
$$

Moreover, (3.10) implies

$$
-\left(\alpha u_{1}-\alpha u_{2}, v_{\varepsilon}\right)_{0, \Sigma}+C_{0}\left\|\nabla v_{\varepsilon}\right\|_{0, Q}^{2} \leq\left(\left(\mathcal{A}\left(u_{1}\right)-\mathcal{A}\left(u_{2}\right)\right) \nabla u_{2}, \nabla v_{\varepsilon}\right)_{0, Q} .
$$

Using Friedrichs' inequality (2.10) and relations (3.13), (3.6), (3.14), (3.11), and (3.12), we obtain

$$
\begin{aligned}
\left\|v_{\varepsilon}\right\|_{0, Q}^{2} & \leq C_{1} \int_{0}^{T}\left(\left\|v_{\varepsilon}\right\|_{0, \Gamma}^{2}+\left\|\nabla v_{\varepsilon}\right\|_{0, \Omega}^{2}\right) d t \\
& \leq C_{2} \int_{0}^{T}\left(\alpha u_{2}-\alpha u_{1}, v_{\varepsilon}\right)_{0, \Gamma} d t+\left\|\nabla v_{\varepsilon}\right\|_{0, Q}^{2} \\
& \leq C_{3}\left(\left(\alpha u_{2}-\alpha u_{1}, v_{\varepsilon}\right)_{0, \Sigma}+C_{0}\left\|\nabla v_{\varepsilon}\right\|_{0, Q}^{2}\right)
\end{aligned}
$$




$$
\begin{aligned}
& \leq C_{3}\left(\left(\mathcal{A}\left(u_{1}\right)-\mathcal{A}\left(u_{2}\right)\right) \nabla u_{2}, \nabla v_{\varepsilon}\right)_{0, Q} \\
& \leq \varepsilon C_{4}\left\|\nabla u_{2}\right\|_{0, Q_{0} \backslash Q_{\varepsilon}}\left\|\nabla v_{\varepsilon}\right\|_{0, Q} \leq \varepsilon^{2} C_{5}\left\|\nabla u_{2}\right\|_{0, Q_{0} \backslash Q_{\varepsilon}}^{2} .
\end{aligned}
$$

We now show that

$$
\operatorname{meas}\left(Q_{0} \backslash Q_{\varepsilon}\right) \rightarrow 0 \quad \text { as } \varepsilon \rightarrow 0 .
$$

Note that

$$
\lim _{\varepsilon \rightarrow 0} \operatorname{meas}\left(Q_{0} \backslash Q_{\varepsilon}\right)=\operatorname{meas} \bigcap_{\varepsilon>0}\left(Q_{0} \backslash Q_{\varepsilon}\right),
$$

because $Q_{0} \backslash Q_{\varepsilon_{1}} \subset Q_{0} \backslash Q_{\varepsilon_{2}}$ whenever $0<\varepsilon_{1}<\varepsilon_{2}$. Suppose, to the contrary, that (3.16) is not valid. Then there exists a set $\widetilde{Q}$ such that meas $\widetilde{Q}>0$ and $\widetilde{Q} \subset Q_{0} \backslash Q_{\varepsilon}$ for all $\varepsilon>0$. We can put, for example, $\widetilde{Q}=\bigcap_{\varepsilon>0}\left(Q_{0} \backslash Q_{\varepsilon}\right)$. We have $\widetilde{Q} \not \subset Q_{\varepsilon}$ for all $\varepsilon>0$, and therefore

$$
\int_{\widetilde{Q}}\left(u_{2}(x, t)-u_{1}(x, t)\right) d x d t \leq \varepsilon \text { meas } \widetilde{Q}
$$

for all $\varepsilon>0$. Thus, the only possibility is

$$
\int_{\widetilde{Q}}\left(u_{2}(x, t)-u_{1}(x, t)\right) d x d t \leq 0 .
$$

On the other hand the inclusion $\widetilde{Q} \subset Q_{0}$ implies, by the definition of $Q_{0}$, that

$$
\int_{\widetilde{Q}}\left(u_{2}(x, t)-u_{1}(x, t)\right) d x d t>0,
$$

which contradicts (3.17), and thus proves (3.16).

Due to estimate (3.15), we arrive at

$$
\begin{aligned}
\operatorname{meas} Q_{\varepsilon} & =\varepsilon^{-2} \int_{Q_{\varepsilon}} \varepsilon^{2} d x d t=\varepsilon^{-2} \int_{Q_{\varepsilon}} v_{\varepsilon}^{2} d x d t \leq \varepsilon^{-2}\left\|v_{\varepsilon}\right\|_{0, Q}^{2} \\
& \leq C_{5}\left\|\nabla u_{2}\right\|_{0, Q_{0} \backslash Q_{\varepsilon}}^{2} \rightarrow 0 \quad \text { as } \varepsilon \rightarrow 0,
\end{aligned}
$$

by $(3.16)$.

Since meas $Q_{0}=$ meas $Q_{\varepsilon}+\operatorname{meas}\left(Q_{0} \backslash Q_{\varepsilon}\right)$, from (3.16) and (3.18) we obtain meas $Q_{0}=0$, which contradicts (3.4).

\section{Conclusions}

Corollary 4.1. There exists at most one weak solution of problem (2.7).

Proof. If $u_{1}$ and $u_{2}$ are two solutions of (2.7) then $u_{1} \leq u_{2}$ and $u_{1} \geq u_{2}$ due to Theorem 3.1. Therefore, $u_{1}=u_{2}$ almost everywhere in $Q$.

We see that the comparison principle easily implies the uniqueness of the solution of our problem. The comparison principle is much more general then the uniqueness theorem itself. It has several applications. For instance, we 
can employ it for testing numerical methods. We know that zero data yield the zero solution. Thus, nonnegative data have to give us a nonnegative solution. If we have in mind some numerical scheme it is natural to define the following property. We say that a numerical method conserves nonnegativity if all nonnegative data give us a nonnegative numerical solution. We note that not all numerical schemes have this property. For more details about positivity conservation see [2]. A similar idea is presented in [7], where a discrete maximum principle is studied in the context of the finite element method.

Acknowledgements. This paper was supported by grant no. 201/01/ 1200 of the Grant Agency of the Czech Republic. This support is gratefully acknowledged.

\section{References}

[1] M. Artola, Sur une classe de problèmes paraboliques quasi-linéaires, Boll. Un. Mat. Ital. B 5 (1986), 51-70.

[2] I. Faragó and R. Horváth, On the nonnegativity conservation of a finite element solution of parabolic problems, in: Finite Element Methods, Three-Dimensional Problems, GAKUTO Internat. Ser. Math. Sci. Appl. 15, 2001, 78-86.

[3] S. Fučík and A. Kufner, Nonlinear Differential Equations, Elsevier, Amsterdam, 1980 .

[4] H. Gajewski, K. Gröger and K. Zacharias, Nichtlineare Operatorgleichungen und Operatordifferentialgleichungen, Akademie-Verlag, Berlin, 1978.

[5] D. Gilbarg and N. S. Trudinger, Elliptic Partial Differential Equations of Second Order, Springer, Berlin, 1977.

[6] I. Hlaváček, M. Křižek and J. Malý, On Galerkin approximations of a quasilinear nonpotential elliptic problem of a nonmonotone type, J. Math. Anal. Appl. 184 (1994), 168-189.

[7] S. Korotov, M. Křižž and P. Neittaanmäki, Weakened acute type condition for tetrahedral triangulations and the discrete maximum principle, Math. Comp. 70 (2000), 107-119.

[8] M. Kř́žžek and L. Liu, On a comparison principle for a quasilinear elliptic boundary value problem of a nonmonotone type, Appl. Math. (Warsaw) 24 (1996), 97-107.

[9] J. L. Lions, Équations différentielles opérationnelles, Springer, 1963.

[10] M. H. Protter and H. F. Weinberger, Maximum Principles in Differential Equations, Prentice-Hall, Englewood Cliffs, NJ, 1967.

[11] T. Vejchodský, On the nonmonotony of nonlinear elliptic operators in divergent form, preprint, Math. Inst., Acad. Sci., Praha, 2001.

Mathematical Institute

Academy of Sciences

Žitná 25

CZ-11567, Praha 1, Czech Republic

E-mail: vejchod@math.cas.cz 